\title{
EL IMPACTO DE LA EDUCACIÓN EN LA INTENCIÓN EMPRENDEDORA DE LOS ESTUDIANTES DEL GRADO DE ECONOMÍA
}

\section{THE IMPACT OF EDUCATION ON THE ENTREPRENEURIAL INTENTION OF STUDENTS IN THE BACHELOR OF ECONOMICS}

Sanchez Torné, Isadora (Universidad de Sevilla) *

Pérez Suárez, Macarena (Universidad de Sevilla) ${ }^{* *}$

\section{Resumen}

Este trabajo tuvo como objetivo conocer la incidencia de la formación sobre Creación de Empresas, una asignatura obligatoria, en estudiantes del grado en Economía desde una perspectiva de género. Con tal fin, se analizará las intenciones, actitudes y capacidades emprendedoras del alumnado que asistió a la citada asignatura durante cinco cursos académicos (un total de 272 respuestas). Bajo un método científico inductivo se observó a la misma población antes y después de la formación. Consecuentemente, se aplicó una serie de pruebas estadísticas para conocer si existen diferencias significativas ex-ante y ex-post formación. Como resultado se identificó el perfil estándar del alumnado de cuarto curso del grado de Economía de la Universidad de Sevilla (mujer con edad media de 22 años), así como una serie de premisas sobre pretensiones, actitudes y capacidades emprendedoras. Las principales derivaciones fueron que aumenta la proporción de personas estudiantes que declara tener una idea para crear una empresa, mejora significativamente la valoración de los conocimientos para crear y dirigir una empresa, además de muchas de las competencias transversales. Sin embargo, la intención y actitud emprendedora se redujo significativamente. Por último, se observó que la educación emprendedora tuvo un mayor efecto en las mujeres que en los hombres, concretamente, ellas aumentaron su valoración en más capacidades empresariales y comportamiento percibido, a pesar de que la intención emprendedora fue superior en los alumnos. La educación emprendedora es un medio de reducir esa diferencia debiendo incorporar modelos de referencia femeninos.

Palabras clave: Educación Superior, Estudiantes, Intención Emprendedora, Género.

JEL: A23, I23, L26, M20.

\section{Abstract}

The aim of this work was to find out the impact of training on the creation of companies, a required subject, on undergraduate students in economics from a gender perspective. To this end the intentions, attitudes and entrepreneurial abilities of the students who attended this subject during five academic years (272 responses) were analysed. Under an inductive scientific method (inferences), the same population was observed before and after the training. Consequently, a series of statistical tests were applied to find out whether there are significant differences ex-ante and ex-post training. As a result, the standard profile of the

* Universidad de Sevilla. isanchez6@us.es.

** Universidad de Sevilla. mperez32@us.es.

Recibido: 30 de diciembre de 2018. Aceptado: 20 de mayo de 2019. 
fourth-year students of the economics degree at the University of Seville (woman with an average age of 22 years) was identified, as well as several premises regarding pretensions, attitudes and entrepreneurial abilities. The main derivations were that: there is an increase in the proportion of students who declare to have an idea to create a company; it significantly improves the valuation of the knowledge to create and manage a company, in addition to many of the transversal competences. However, the entrepreneurial intention and attitude was significantly reduced. Finally, it was observed that entrepreneurial education had a greater effect on women than on men, specifically; they increased their value in more entrepreneurial skills and perceived behaviour, despite the fact that the entrepreneurial intention was higher in man students. Entrepreneurial education is a means of reducing this difference by incorporating female reference models.

Keywords: Higher Education, Students, Entrepreneurial Intent, Gender.

JEL: A23, I23, L26, M20.

\section{INTRODUCCIÓN}

El emprendimiento se ha revelado como un factor clave para el crecimiento económico, el aumento de la competitividad y la fuente de nuevos yacimientos de empleos en las regiones (Comisión Europea, 2012). De ahí que diversas autorías investiguen los elementos que lo fomentan, y que cobre especial importancia en la situación actual de crisis económica, al percibirse como un medio de reactivación sostenible (Oliver et al., 2016). El emprendimiento es una vía de inserción laboral ante las altas tasas de desempleo (Sanchis-Palacio y RedondoCano, 1997; Martín et al,. 2013).

Las instituciones son conscientes de los beneficios del emprendimiento y la necesidad de potenciarlo. En tal reconocimiento, la Unión Europea ha aprobado diferentes programas y proyectos como el Plan de Acción sobre Emprendimiento 2020 (Comisión Europea, 2013) que propone, entre otras medidas, el fomento de la educación emprendedora en todos los niveles educativos y dinamizar la cultura emprendedora. A nivel nacional, destaca la Estrategia Juventud 2020 aprobada en el Consejo de Ministros en España el 12 de septiembre de $2014^{1}$ que junto a la Estrategia de Emprendimiento y Empleo Joven 2017-2020² abordan, además de otras medias de fomento, la inclusión de la educación emprendedora en los diferentes niveles de enseñanza. A nivel autonómico, existen las iniciativas canalizadas a través de los Centros de Apoyo al Desarrollo Empresarial ${ }^{3}$.

De igual manera, sobre la relación comunidad y Universidad, aparecen nuevos cometidos institucionales hasta llegar a poner en circulación el término «universidades emprendedoras» (Davis y Diamond, 1997; Etzkowitz y Dzisah, 2008). Según Liñán y Fayolle (2015), las universidades son una de las instituciones más relevantes junto con las redes sociales representantes de los empresarios, la cultura y contexto regional. También aparece la reivindicación de una mayor implicación en el entorno, a través de la intervención educativa, para incrementar el conocimiento y la capacidad empresarial. Jaén y Liñán (2013) sostienen que un ambiente institucional favorable hacia el emprendimiento implicaría una valoración más positiva de las iniciativas empresariales y mejoraría su reconocimiento social. En palabras de Salinas y Osorio (2012, p.131), "para que el emprendimiento genere no solo empleo e ingresos sino también innovación, se requiere la convergencia e integración «cuatripartita» entre Universidad, Estado, Comunidad y Empresa”. Mientras que Urbano y

\footnotetext{
${ }^{1}$ Fuente: http://www.lamoncloa.gob.es/consejodeministros/referencias/Paginas/2014/refc20140912.aspx (Consultado 31.03.2018).

${ }^{2}$ Fuente: http://www.mineco.gob.es/stfls/mineco/comun/pdf/170503_np_reformas.pdf (Consultado 31.03.2018).

${ }^{3}$ Fuente: https://www.andaluciaemprende.es/CADE/ (Consultado 31.03.2018).
} 
Guerrero (2013) apuntan que es vital, y más aún en tiempos de crisis, el trabajo conjunto de potenciar el emprendimiento para reactivar la economía y garantizar la riqueza social.

Valencia et al. (2016) ponen de relieve que parte del éxito empresarial está vinculado al capital humano, por lo que la educación se configura como elemento principal para el desarrollo del emprendimiento. En esta línea, según Salinas y Osorio (2012), la universidad es el centro adecuado para incentivar el espíritu emprendedor y formar en las capacidades emprendedoras. A tenor de estas evidencias, según Ruiz et al. (2014), las investigaciones han ido desde medir la incidencia de la educación para el emprendimiento en la posible intención futura empresarial a estipular los enfoques, contenidos y metodologías más adecuadas para desarrollar la intención emprendedora.

Por otra parte, numerosas exploraciones han utilizado a estudiantes universitarios, tanto de titulaciones relacionadas con la rama de economía como de otras, para analizar sus intenciones emprendedoras. El uso de población estudiantil universitaria ha sido avalado por diversos autores (Ruiz et al., 2014; Valencia, et al., 2016; Fernández-Cornejo et al., 2016, Caro-González et al., 2018). Concretamente, Krueger (1993) afirmó que en el análisis de la actitud empresarial existen similitud entre una población de estudiantes y no estudiantes. Sánchez-García et al. (2005) consideran que el alumnado es una población potencialmente emprendedora, además, de ser representativos de la sociedad para analizar el comportamiento humano (Harrison y List, 2004). Por otro lado, Goyanes (2015) opina que el estudio de los propósitos emprendedores de la población joven implicará alcanzar una mayor comprensión sobre los factores que influyen en dichas intenciones. Asimismo, si esta población universitaria corresponde a último curso debe estar más preocupada por su inserción laboral que aquellos que se encuentran en las primeras etapas de su educación terciaria (Lemmink et al., 2003).

El estado vigente de la intención emprendedora de los estudiantes universitarios españoles fue analizado por Guerrero et al. (2016). Este estudio está compuesto por preguntas de valoración (Escala 1 a 7, siendo 7 el mayor valor) y respuesta múltiple (resultados en porcentaje). Los principales resultados para el alumnado de cuarto curso son: 57,3 por ciento indicó tener deseo de crear una empresa, pero solo el 33 por cien mostró intención de iniciar una actividad emprendedora en los próximos 3 años; la actitud emprendedora tuvo una valoración de 4; 50 por cien expresó que el miedo al fracaso era uno de los motivos para no iniciar un negocio; 29,3 por cien tuvo percepción de poseer los conocimientos y habilidades necesarios para emprender (valoración media del 3,16). En relación al entorno universitario, consideraron que los centros de estudio no promueven suficiente el emprendimiento (valoración media del 3,57). Sobre la valoración de las competencias emprendedoras que desarrollan las asignaturas los resultados fueron los siguientes: 5,10 en competencias emprendedoras interpersonales (trabajo en equipo, desarrollo de red de contacto, cooperación, ética); 4,27 en competencias emprendedoras sistemáticas (identificar oportunidades de negocio, creatividad, administrar, innovación); 4,94 en competencias emprendedoras instrumentales (resolución de problemas, toma de decisiones, competencias técnicas/financieras).

A nivel macro, se hallan los informes territoriales como el GEM ${ }^{4}$, Global Entrepreneurship Monitor. Concretamente, en diferentes comunidades autónomas se han elaborado informes sobre la intención emprendedora de sus estudiantes universitarios, para el caso de Andalucía por ESIC Andalucía (2018). En él se indica que: el 36,6 por ciento desea

${ }^{4}$ Fuente: $\quad$ España $\quad$ http://www.gem-spain.com/wp-content/uploads/2018/09/PDF-17-1695-9302.pdf; Andalucía http://www.gem-spain.com/wp-content/uploads/2015/03/Informe-GEM-Andalucia-2016.pdf (Consultado 18.12.2018). 
tener un negocio por cuenta propia, entre las principales motivaciones para emprender destaca el reto personal y, en relación a los obstáculos se hace espacial énfasis en el riesgo y miedo al fracaso.

En el binomio intención emprendedora y género diversas investigaciones han abordado las diferencias entre mujeres y hombres, poniendo de manifiesto una controversia en la incidencia de la educación emprendedora (Bae et al., 2014).

Una connotación de lo argumentado es el objetivo de este artículo, que comparte propósito con Ruiz et al. (2014), al comprobar el impacto de la educación sobre el emprendimiento y el posible sesgo por género en una población de último curso del grado en Economía (272 estudiantes) bajo la observación «antes» y «después» de recibir la formación. Para ello, tal como se explica en el apartado de metodología, se suministró una encuesta (a la misma población antes y después de la formación) que ha sido validada previamente.

Esta investigación se estructura en cuatro partes, la primera dedicada a realizar una revisión de la literatura de los resultados sobre el impacto de la educación emprendedora (en estudiantes universitarios tanto en su conjunto como por género) sobre la intención de emprender, actitud hacia el emprendimiento, comportamiento percibido y capacidades. Seguidamente, se explica la metodología para analizar los datos. En tercer lugar, se exponen los resultados obtenidos, y, por último, las conclusiones halladas que dan respuestas a las hipótesis planteadas. En definitiva, esta investigación viene a sumar valor en la creación de conocimientos y en el tratamiento de una realidad específica y singular, que al mismo tiempo que proporciona información a diversas partes involucradas del ámbito académico local y regional, insta a trabajar en exploraciones científicas más ambiciosas. Todo ello, sin duda, bajo la creencia de que la universidad ha de investigar para crear sociedad, junto al reconocimiento, al igual que considerables expertos, que es clave involucrar a las personas docentes en indagaciones sujetas a la innovación y llevar los resultados a las aulas para que el alumnado participe en el cambio de paradigma educativo (Lerís y Sein-Echaluce, 2011).

\section{REVISIÓN DE LA LITERATURA}

En el propósito de evidenciar el impacto de la educación sobre el emprendimiento y el posible sesgo por género en una población universitaria, se plantea un estado del arte a partir de varios temas: educación empresarial, impacto de la educación superior, intención y comportamiento emprendedor, y género.

Son múltiples las investigaciones que analizan la intención emprendedora de los estudiantes universitarios como predictor del comportamiento futuro (Krueger y Brazeal, 1994), Proyecto GUESSS (Sieger et al., 2016). Junto a las investigaciones que miden el impacto de la educación emprendedora en la intención emprendedora (Abaho et al., 2015; Bell, 2015; Boukamcha, 2015; Brink y Madsen, 2015; Fayolle y Gailly, 2015; Piperopoulos y Dimov, 2015; Rauch y Hulsink, 2015; Entrialgo e Iglesias, 2016), pues sus hallazgos muestran la incidencia significativa de la educación empresarial, al mismo tiempo que son relevantes al centrar el tema objeto de estudio. De forma infalible, Baptista y Naia (2015) y Nabi et al. (2017) examinan al detalle el estado la literatura integral sobre la relación educación empresarial e intención emprendedora.

Una vez reconocidos los estudios de referencia, se procede a la identificación teórica. Para Ruiz et al. (2014, p. 84), “de entre los modelos teóricos desarrollados para explicar la intención emprendedora, dos han recibido especial atención: el modelo del evento emprendedor (Shapero y Sokol, 1982) y la teoría de la acción planificada (Ajzen, 1991; 2001)”. 
El presente trabajo toma de referencia el modelo del evento emprendedor de Shapero y Sokol (1982), conforme al mismo, la elección del sujeto para iniciar una nueva empresa depende de tres conjuntos de elementos: la percepción de deseabilidad ${ }^{5}$, la percepción de viabilidad y la propensión a actuar. Los cuales equivalen a la actitud personal ante el emprendimiento, control del comportamiento percibido e intención emprendedora. Por su parte, el modelo de Ajzen sobre intención emprendedora se basa en la teoría de la conducta planificada cuyo fundamento principal reside en que el comportamiento humano requiere de cierta cantidad de planificación precedente, y que, en dicha planificación, así como en su comportamiento ulterior, le influyen las creencias. Unas creencias que afectan directamente a las intenciones que son planificadas en base a dos factores: la actitud hacia la acción y las normas subjetivas.

No existe consenso sobre el efecto de la educación empresarial en la intención de emprender. Autores como Oosterbeek et al. (2010); Ruiz et al. (2014); Lima et al. (2015) y Popescu et al. (2016) no obtuvieron evidencias de que la formación en emprendimiento aumentara la intención emprendedora. Mientras que Maresch et al. (2016) sí hallaron resultados positivos y significativos, aunque bastante bajos; al igual que Ndofirepi y Rambe (2017), aseverando que la educación emprendedora afectó en los elementos influyentes en la intención emprendedora, pero sin cambios reveladores. En cambio, otros estudios (Hannon, 2006; Jones et al., 2008; Wilson et al., 2009; Lanero et al., 2011; Jiang et al., 2017; SoriaBarreto et al., 2017) encontraron una relación positiva entre educación emprendedora e incremento de las intenciones emprendedoras.

Hipótesis 1: La educación emprendedora tiene un efecto positivo en la intención de emprender del alumnado.

Ajzen (2005) afirmó que la intención para desarrollar un cierto comportamiento se transforma en acción cuando llega el momento adecuado. Así que la actitud personal ante el emprendimiento, definida como el grado en que una persona tiene una valoración favorable o desfavorable a una conducta emprendedora (Valencia et al., 2014), cobra gran relevancia en el estudio intencional. Los autores Shahverdi et al. (2018), indicaron que la formación en emprendimiento ayuda a reducir las barreras percibidas a la hora de emprender. Por último, Nabi et al. (2018) realizaron una reflexión sobre estos dos tipos de resultado, concluyendo que en los casos donde la educación emprendedora eleva la intención emprendedora estaba aparejada la acumulación de experiencias de aprendizaje positivo, trabajos prácticos e inspiración por sus docentes o compañeros. En el lado opuesto, se hallaron personas que habían desarrollado una perspectiva más realista del proceso de emprendimiento, entendiendo su complejidad y los desafíos que conlleva.

Hipótesis 2: La educación emprendedora tiene un efecto positivo en la actitud personal ante el emprendimiento.

En consonancia con Krueger et al. (2000), el control del comportamiento percibido refleja la viabilidad percibida para realizar un comportamiento, por lo que se relaciona con la percepción que tiene un sujeto de sus propias competencias para afrontar una situación (autoeficacia). Lanero et al. (2011), Nizam Zainuddin et al. (2012), Díaz-García et al. (2015), Sánchez-García y Hernández-Sánchez (2016) observan evidencias de que la educación tuvo

\footnotetext{
${ }^{5}$ Krueger y Brazeal (1994) matizan el modelo de Shapero y Sokol, “incorporando aspectos de Ajzen, y lo denominan modelo del potencial emprendedor, donde se integran todos los conceptos de percepción de viabilidad y deseabilidad, así como la propensión a actuar y el suceso disparador (...) En este modelo se destaca la relación de las percepciones de deseabilidad y de viabilidad como factores determinantes de la credibilidad emprendedora. Se define la percepción de deseabilidad como lo más o menos atractivo que resulta para la persona el hecho de poner en marcha un negocio. Por lo tanto, estas actitudes personales derivarán en posibles resultados, más o menos favorables, atribuibles a la acción” (Mussons-Torras y TarratsPons, 2018, p. 297).
} 
un efecto positivo en la percepción de la viabilidad del emprendimiento, que a su vez afectó en el control del comportamiento percibido. En cambio, Pihkala (2008) halló que la educación emprendedora disminuyó la autoeficacia empresarial lo que repercutió negativamente en el control del comportamiento percibido. Lima et al. (2015) ofreciera una posible justificación a este tipo de resultados, alegando que los estudiantes que recibieron formación emprendedora son más conscientes de las necesidades que demanda iniciar y gestionar una empresa. Zhao et al. (2005) sostienen que las personas con elevada intención emprendedora fueron aquellas que también poseían altos niveles de autoeficacia empresarial, es decir, individuos que consideraban que tendrían éxito como empresarios.

Hipótesis 3: La educación emprendedora aumenta la valoración del control del comportamiento percibido.

Las características emprendedoras son habilidades generales, aplicables en todos los campos de la vida (Joensuu-Salo et al., 2015), entre las que destacan: toma de decisión, generar nuevas ideas, aportar solución, espíritu crítico, trabajo en equipo y motivar al equipo de trabajo.

Hipótesis 4: La educación emprendedora desarrolla capacidades claves para el emprendimiento.

Ruiz et al. (2014) refieren la influencia del factor de género, mostrada como relevante en la intención de emprender. Siendo múltiples los factores de naturaleza psicosocial los que contribuyen a explicar la disparidad de género respecto a los intereses y comportamientos asociados al emprendimiento.

Específicamente, son diversas las evidencias obtenidas por los investigadores en cuanto a los diferentes efectos de la educación emprendedora en función del género (Wilson et al., 2007; Ruiz-Navarro et al., 2012; Medina et al., 2014; Ruiz et al., 2014; Westhead y Solesvik, 2016; Soria-Barreto et al., 2017). Por su parte, Popescu et al. (2016) y Soria-Barreto et al. (2017) observaron una respuesta similar entre hombres y mujeres en relación a la intención emprendedora, pero Jones et al. (2008) indicaron que las mujeres experimentaron un aumento de la intención emprendedora superior a los hombres. Tanto Zhao (2005), Ruiz et al. (2014), como Wilson et al. (2009) concluyeron que las mujeres manifestaron una autoeficacia menor, pero los primeros sí hallaron un incremento tras la educación emprendedora, y los últimos no. En contraposición, Medina et al. (2014) señalan que los hombres exhiben una mayor vocación emprendedora que las mujeres y Fuentes-García y Sánchez-Cañizares (2010) una menor iniciativa emprendedora en las mujeres. Wilson et al. (2007) y Soria-Barreto et al. (2017) evidenciaron que la educación emprendedora tiene efectos diferentes en hombres y mujeres, repercutiendo cambios más profundos en ellas, hallando una correlación significativa y leve entre la educación empresarial e intenciones empresariales, el género de los sujetos participantes es una variable moderadora (Camelo-Ordaz et al., 2016; Entrialgo e Iglesias, 2016). Así que "el impacto de la educación emprendedora en la intención emprendedora puede no ser tan efectivo para hombres como para mujeres” (Bae et al., 2014, p. 223).

Hipótesis 5: La educación emprendedora exhibe diferencias de género, pues tiene un efecto superior en las mujeres que en los hombres.

Tras platear las cinco hipótesis se vuelve a evidenciar la carencia de investigaciones centradas en medir el impacto de la formación emprendedora (controlando la población de inicio y final), y más concretamente, aquellas dirigidas a evaluar las diferencias entre hombres y mujeres. A continuación, se detalla la metodología empleada para contrastar las hipótesis anteriormente enunciadas. 


\section{METODOLOGÍA}

La población objeto de análisis es el alumnado de la asignatura de «Creación de Empresas» (6 créditos ECTS) del Grado de Economía de la Universidad de Sevilla, que se imparte en el primer cuatrimestre de cuarto curso. Con ella, el profesorado pretende que estudiantes desarrollen un proyecto empresarial real y completo, bajo el formato de proyectos colaborativos, y con la finalidad de provocar capacidad y cultura emprendedora. De tal forma que acceden al procedimiento de desarrollo de un proyecto empresarial, en primera persona, y conocen que emprender no implica asumir riesgos sino aprender a controlarlos.

La metodología empleada en la formación de la población estudiantil de la asignatura de «Creación de Empresas» fue inductiva en su conjunto, primando el enfoque de investigaciónacción (Romera-Iruela, 2011) en un proceso de enseñanza-aprendizaje (Moreira, 1993; Naves Cappelle et al., 2012). Es por ello, que se utilizara proyectos dentro del currículo, es decir, el Aprendizaje Basado en Proyectos (Márquez y Jiménez-Rodrigo, 2014; Wiek et al., 2014; Carrasco et al., 2015; Savery, 2015) ${ }^{6}$.

El estudio se centra en la población estudiantil que cursó la citada asignatura durante cinco cursos académicos, 2012-13, 2013-14, 2014-15, 2015-16, 2016-17 (42, 46, 32, 64 у 88 personas, respectivamente). La recogida de información versa sobre una encuesta suministrada en papel en dos momentos: al inicio y al final del periodo formativo. Siendo observada la misma población antes y después de la formación, es decir, solo se han seleccionado los cuestionarios correspondientes a las personas estudiantes que respondieron en los dos momentos (Sánchez-García, 2011; Fayolle y Gailly, 2015), cantidad que ascendió a 272. Esto ha permitido realizar un análisis especialmente singular, por el que se puede conocer cómo han evolucionado las intenciones emprendedoras, capacidades y competencia en una misma población antes y después de recibir la formación emprendedora. Debido al nivel de implicación necesaria por parte del alumnado para superar esta asignatura, se reconoce que todos los estudiantes han sido afectados en mayor o menor medida por la educación emprendedora impartida. Asimismo, es preciso decir que no se llevó a cabo un control de las variables externas a la formación que pudieran influir en el alumnado, por ello partimos de la premisa céteris páribus.

La encuesta se basó en las investigaciones de Liñán y Chen (2009) sobre la modelización de las variables adecuadas para pedir la intención emprendedora. Inicialmente se formulan preguntas de carácter dicotómico (Si/No) y abierto, ambas dirigidas a conocer los aspectos de registro (edad, sexo, residencia) y la deseabilidad del alumnado por crear una empresa. El último bloque está destinado a estudiar las actitudes emprendedoras y competencias, a partir de preguntas de valoración siguiendo una escala Likert de cinco posiciones, donde 1 significa totalmente desacuerdo y 5 totalmente de acuerdo. Con ello, se pretendió conocer la actitud personal, comportamiento percibido, intención y capacidades para emprender del alumnado. Dichas cuestiones han sido usadas por otros investigadores para determinar la intención emprendedoras estudiantil como, por ejemplo: Liñán et al. (2011); Liñán et al. (2011); Sahinidis et al. (2012); Olguín y Caro-González (2015); González et al. (2016); Srivastava y Misra (2017).

Particularizar que, a diferencia de autorías como Liñán et al. (2016), no se estudia la variable norma social. Esto se debe a dos motivos, primeramente, el presente trabajo se basa en las investigaciones de Shapero y Sokol (1982), los cuales no contemplan la norma social,

\footnotetext{
${ }^{6}$ Según expertos de WISE España (2015) -Cumbre Mundial por la Innovación en Educación-, el aprendizaje basado en proyectos es uno de los mayores retos educativos existentes.
} 
por otro lado, diversas investigaciones han evidenciado la poca o nula influencia de ésta en la intención emprendedora (Díaz-Casero et al., 2007 y Sánchez-García, 2010).

La escala utilizada ha sido validada con los estudios precedentes y ha presentado gran consistencia, así mismo, en este caso los resultados de Alpha de Cronbach por bloques de preguntas (ver cuadro 1 ) están por encima de 0,7 lo que se considera un valor aceptable (George y Mallery, 2003, p. 231).

\section{CUADRO 1: PRUEBA ALPHA DE CRONBACH. FIABILIDAD DE LA ESCALA.}

\begin{tabular}{|c|c|}
\hline Bloques de preguntas & Alpha de Cronbach \\
\hline Actitudes personales ante el emprendimiento & 0,854 \\
\hline Comportamiento percibido & 0,848 \\
\hline Intención emprendedora & 0,912 \\
\hline Capacidades & 0,836 \\
\hline
\end{tabular}

Fuente: Elaboración propia.

Previamente al tratamiento estadístico de los resultados, se realizaron dos actuaciones: en primer lugar, eliminar aquellas contestaciones que no hubieran respondido al inicio y al final del periodo formativo. En segundo lugar, pasar las encuestas seleccionadas del papel a Excel. Finalizadas ambas acciones, se procedió a registrar los datos en el programa estadístico SPSS (24V), para su posterior tratamiento estadístico.

Con el fin de comprobar las hipótesis planteadas, se empleó un análisis de inferencia (Usman, 2019). Tales pruebas son las idóneas para hallar diferencias significativas entre grupos, en este caso, los grupos fueron alumnado al inicio de la formación y al finalizar. Concretamente, las pruebas realizadas consistieron en: 1) prueba de Mcnemar para el caso de las preguntas dicotómicas (adecuada para conocer si la formación en emprendimiento induce a un cambio en las respuestas), 2) prueba $T$ para muestras relacionadas. En los cuatro casos, el nivel de confianza fue del 95 por cien y el error del 5 por cien. Es preciso aclarar que para estos cálculos no se tuvo en cuenta las respuestas Ns/Nc, por ese motivo los totales no coinciden en todos los cuadros.

Las variables de actitud personal, comportamiento percibido, intención y capacidades para emprender del alumnado se midieron a través de un conjunto de preguntas formuladas al alumnado, las cuales se recogen en el cuadro 2. El análisis de inferencia consideró las medias agregadas de cada apartado, con lo que resultó una única variable para cada bloque. 


\section{CUADRO 2: PREGUNTAS QUE COMPONEN LAS VARIABLES DE ACTITUD PERSONAL ANTE EL EMPRENDIMIENTO, CONTROL DE COMPORTAMIENTO PERCIBIDO, INTENCIÓN EMPRENDEDORA Y CAPACIDADES.}

\begin{tabular}{|l|}
\hline Actitudes personales ante el emprendimiento \\
\hline 1.Ser una persona emprendedora tiene más ventajas que inconvenientes (ap1) \\
\hline 2.Ser una persona emprendedora me resulta atractivo (ap2) \\
\hline 3.Si tuviese la oportunidad y los medios me gustaría crear mi propia empresa (ap3) \\
\hline 4.Convertirme en persona emprendedora sería algo que me dejaría muy satisfecho (ap4) \\
\hline 5.De entre varias opciones, preferiría ser persona emprendedora (ap5) \\
\hline Control del comportamiento percibido \\
\hline 6.Crear una empresa y mantenerla en funcionamiento sería fácil para mí (cp6) \\
\hline 7.Estoy capacitado/a para poner en marcha una empresa viable (cp7) \\
\hline 8.Puedo controlar el proceso de creación de una nueva empresa (cp8) \\
\hline 9.Conozco los detalles prácticos necesarios para crear una empresa (cp9) \\
\hline 10.Sé cómo desarrollar un proyecto empresarial (cp10) \\
\hline 11.Si intentase crear una empresa, tendría muchas posibilidades de éxito (cp11) \\
\hline Intención Emprendedora \\
\hline 12.Estoy preparado/a para hacer lo necesario para convertirme en una persona emprendedora (ie12) \\
\hline 13. Mi meta profesional es llegar a ser una persona emprendedora (ie13) \\
\hline 14.Realizaré todos los esfuerzos precisos para iniciar y continuar mi propia empresa (ie14) \\
\hline 15.Estoy decidido/ a crear una empresa en el futuro (ie15) \\
\hline 16.Pienso con bastante firmeza en crear mi propia empresa (ie16) \\
\hline
\end{tabular}
Fuente: Elaboración propia.

\section{RESULTADOS}

\subsection{Principales características de la muestra obtenida}

La muestra la componen 272 estudiantes, donde el 45,59 por cien son hombres y el 54,41 por cien mujeres. El 80 por cien tenía entre 21 y 24 años, la edad media fue de 22 años y la más frecuente 21. La principal provincia de procedencia del alumnado fue Sevilla (55,33 por cien) seguida de Cádiz (23 por cien). A continuación, detallar los principales resultados obtenidos a partir de los principales bloques de la encuesta (cuestiones sobre emprendimiento, actitud ante el emprendimiento y competencias).

\subsection{Deseabilidad del alumnado por tener su propia empresa o negocio}

Se planteó si en algún momento se había tenido deseo de tener su propia empresa o negocio (ver cuadro 3). La probabilidad $(\mathrm{P}=0,004<0,05)$ asociada a la prueba de Mcnemar resultó significativa, de lo que se deduce que ocurrió un cambio significativo en la proporción de alumnado que les gustaría tener su propia empresa o negocio al final de la formación (68,89 por cien) respecto al porcentaje que sí lo hizo al inicio (76,30 por cien) del curso. Se observan diferencias por género, ya que en el caso de las mujeres sí se obtuvo una probabilidad $(\mathrm{P}=0,003<0,05)$ asociada a la prueba de Mcnemar significativa, mientras que en los hombres no fue así $(\mathrm{P}=0,774>0,05)$. Por lo que la educación emprendedora sí afectó a las alumnas, implicando una reducción (inicio 72,79 por ciento y final 60,54 por cien) de deseo de tener su propia empresa. Asimismo, es preciso añadir que los hombres manifestaron un porcentaje significativamente superior a las mujeres en el momento final. 


\section{CUADRO 3: ALUMNADO QUE LE GUSTARÍA TENER SU PROPIA EMPRESA O NEGOCIO.}

\begin{tabular}{|c|c|c|c|c|c|c|}
\hline \multirow{3}{*}{$\begin{array}{l}\text { Te gustaría tener tu } \\
\text { propia empresa o } \\
\text { negocio (inicial) }\end{array}$} & \multicolumn{6}{|c|}{ Te gustaría tener tu propia empresa o negocio (final) } \\
\hline & \multicolumn{2}{|c|}{ No } & \multicolumn{2}{|c|}{ Sí } & \multicolumn{2}{|c|}{ Total } \\
\hline & $\mathrm{N}$ & $\%$ & $\mathrm{~N}$ & $\%$ & $\mathrm{~N}$ & $\%$ \\
\hline \multicolumn{7}{|l|}{ Total } \\
\hline No & 52 & $19,26 \%$ & 12 & $4,44 \%$ & 64 & $23,70 \%$ \\
\hline Sí & 32 & $11,85 \%$ & 174 & $64,44 \%$ & 206 & $76,30 \%$ \\
\hline Total & 84 & $31,11 \%$ & 186 & $68,89 \%$ & 270 & $100 \%$ \\
\hline \multicolumn{7}{|l|}{ Mujeres } \\
\hline No & 33 & $22,45 \%$ & 7 & $4,76 \%$ & 40 & $27,21 \%$ \\
\hline Sí & 25 & $17,01 \%$ & 82 & $55,78 \%$ & 107 & $72,79 \%$ \\
\hline Total & 58 & $39,46 \%$ & 89 & $60,54 \%$ & 147 & $100 \%$ \\
\hline \multicolumn{7}{|l|}{ Hombres } \\
\hline No & 19 & $15,45 \%$ & 5 & $4,07 \%$ & 24 & $19,51 \%$ \\
\hline Sí & 7 & $5,69 \%$ & 92 & $74,80 \%$ & 99 & $80,49 \%$ \\
\hline Total & 26 & $21,14 \%$ & 97 & $78,86 \%$ & 123 & $100 \%$ \\
\hline
\end{tabular}

\subsection{Actitudes ante el Emprendimiento}

En este apartado se pretende conocer las actitudes ante el emprendimiento de los estudiantes, que fueron agrupadas 3 bloques (actitudes personales ante el emprendimiento, comportamiento percibido, intención emprendedora)

La actitud personal ante la idea de crear una empresa (ver cuadro 4) tanto del colectivo en su conjunto como de los hombres y mujeres se redujo después de la formación. Es preciso resaltar que la cuestión más valorada tanto al inicio y al final de la formación es «si tuviese la oportunidad y los medios me gustaría crear mi propia empresa (ítem 3)», esto puede sugerir que uno de los obstáculos para el emprendimiento es la falta de oportunidades y medios para llegar a cabo una iniciativa empresarial.

Debido al aumento de la valoración que manifestaron los alumnos y alumnas sobre su control del comportamiento percibido (ver cuadro 4), se puede considerar que la educación en emprendimiento dotó a los estudiantes de formación suficiente para influir positivamente en la percepción de sus capacidades para crear y dirigir una empresa.

En relación a la intención emprendedora de los estudiantes (ver cuadro 4) no se hallaron diferencias significativas ni para el total de alumnado ni por sexo. Lo cual manifiesta que la educación emprendedora impartida no afectó directamente al propósito de crear una empresa. 


\begin{tabular}{|c|c|c|c|c|c|c|c|}
\hline \multicolumn{8}{|c|}{$\begin{array}{l}\text { CUADRO 4: VALORACIÓN DEL ALUMNADO DE SU ACTITUD PERSONAI } \\
\text { ANTE EL EMPRENDIMIENTO, CONTROL DEL COMPORTAMIENTO } \\
\text { PERCIBIDO E INTENCIÓN EMPRENDEDORA. }\end{array}$} \\
\hline & & \multicolumn{6}{|c|}{ Momento } \\
\hline & & \multicolumn{3}{|c|}{ Inicial } & \multicolumn{3}{|c|}{ Final } \\
\hline & & Media & DT & Moda & Media & DT & Moda \\
\hline \multirow{3}{*}{$\begin{array}{c}\text { Actitudes } \\
\text { personales ante el } \\
\text { emprendimiento }\end{array}$} & Total & $3,69 *$ & 1,02 & 4 & $3,56^{*}$ & 1,10 & 4 \\
\hline & Alumnas & $3,57 *$ & 1,01 & 4 & $3,41^{*}$ & 1,09 & 4 \\
\hline & Alumnos & $3,84 *$ & 1,01 & 4 & $3,75^{*}$ & 1,07 & 4 \\
\hline \multirow{3}{*}{$\begin{array}{c}\text { Control del } \\
\text { comportamiento } \\
\text { percibido }\end{array}$} & Total & $2,76^{*}$ & 0,92 & 3 & $3,21 *$ & 0,93 & 4 \\
\hline & Alumnas & $2,60 *$ & 0,86 & 3 & $3,09 *$ & 0,91 & 3 \\
\hline & Alumnos & $2,94 *$ & 0,96 & 3 & $3,36^{*}$ & 0,94 & 4 \\
\hline \multirow{3}{*}{$\begin{array}{l}\text { Intención } \\
\text { Emprendedora }\end{array}$} & Total & 3,00 & 1,10 & 3 & 2,97 & 1,11 & 3 \\
\hline & Alumnas & 2,77 & 1,04 & 3 & 2,73 & 1,04 & 3 \\
\hline & Alumnos & 3,27 & 1,11 & 3 & 3,26 & 1,12 & 4 \\
\hline \multicolumn{8}{|l|}{${ }^{*}$ p-level $<0,05$} \\
\hline
\end{tabular}

\subsection{Capacidades para Emprender}

Conforme al estudio de las capacidades para emprender se plantearon un conjunto de preguntas recogidas en el cuadro 5. La valoración media del alumnado al finalizar el curso es significativamente superior en el tercer ítem (Soy capaz de resolver prácticamente cualquier problema que se me presente) y los dos últimos ítems (Soy perfectamente capaz de desarrollar nuevos productos y servicios y Soy perfectamente capaz de establecer una red de contactos personales y profesionales). Por ello, se puede suponer que la formación ha potenciado positivamente en la capacidad de resolver todo tipo de problemas, la creatividad y la consideración de las habilidades sociales, y en la destreza de establecer una red de contactos. Se observó que los resultados entre hombres y mujeres son sustancialmente diferentes. La formación en emprendimiento implicó un aumento significativo de la valoración de las capacidades de las mujeres, pero no de los hombres.

\section{CUADRO 5: VALORACIÓN DEL ALUMNADO DE SUS PROPIAS CAPACIDADES PARA EMPRENDER.}

\begin{tabular}{|c|c|c|c|c|c|c|}
\hline \multirow{3}{*}{ Capacidades } & \multicolumn{6}{|c|}{ Momento } \\
\hline & \multicolumn{3}{|c|}{ Inicial } & \multicolumn{3}{|c|}{ Final } \\
\hline & Media & DT & Moda & Media & DT & Moda \\
\hline Media del bloque del total del alumnado & $3,40 *$ & 0,90 & 4 & $3,53 *$ & 0,90 & 4 \\
\hline $\begin{array}{l}\begin{array}{l}\text { Soy perfectamente capaz de } \\
\text { oportunidades. }\end{array} \\
\end{array}$ & 3,28 & 0,85 & 3 & 3,34 & 0,88 & 4 \\
\hline Soy muy creativo/a & 3,18 & 0,99 & 3 & 3,27 & 0,98 & 4 \\
\hline $\begin{array}{l}\text { Soy capaz de resolver prácticamente cualquier } \\
\text { problema que se me presente }\end{array}$ & $3,46^{*}$ & 0,83 & 4 & $3,58^{*}$ & 0,82 & 4 \\
\hline $\begin{array}{l}\text { Soy capaz de dirigir a otras personas y de } \\
\text { comunicarles adecuadamente mis ideas e intenciones }\end{array}$ & 3,85 & 0,85 & 4 & 3,87 & 0,83 & 4 \\
\hline $\begin{array}{l}\text { Soy perfectamente capaz de desarrollar nuevos } \\
\text { productos y servicios }\end{array}$ & $3,20 *$ & 0,80 & 3 & $3,42^{*}$ & 0,85 & 3 \\
\hline $\begin{array}{l}\text { Soy perfectamente capaz de establecer una red de } \\
\text { contactos personales y profesionales }\end{array}$ & $3,45^{*}$ & 0,91 & 4 & $3,67^{*}$ & 0,90 & 4 \\
\hline Media de las capacidades de las alumnas & $3,22 *$ & 0,86 & 3,00 & $3,42 *$ & 0,87 & 4,00 \\
\hline Media de las capacidades de los alumnos & 3,62 & 0,89 & 4,00 & 3,65 & 0,92 & 4,00 \\
\hline \multicolumn{7}{|l|}{$*$ p-level<0,05 } \\
\hline
\end{tabular}




\section{CONCLUSIONES}

El objetivo de este artículo fue comprobar el impacto de la educación sobre emprendimiento y el posible sesgo por género en una población de último curso del grado en Economía, antes y después de recibir la formación. Para ello, se realizó un análisis descriptivo e inferencial, de los datos extraídos de una encuesta contestada por el alumnado, que permitió verificar las cinco hipótesis planteadas. A continuación, se detallan las conclusiones más relevantes que ofrecen los resultados.

Las primeras derivaciones que se han obtenido es que la Hipótesis 1 (la educación emprendedora tiene un efecto positivo en la intención de emprender del alumnado) no se sostiene. Se debe a que el porcentaje de personas estudiantes que afirmaron que les gustaría tener su propio negocio o empresa al finalizar la formación se redujo (76,30 por cien ex-antes, 68,89 por cien ex-post). Además, la valoración del alumnado sobre la intención emprendedora no varió antes y después de la formación (3,00 ex-antes, 2,97 ex-post). Por ello, se coincide con las autorías que no obtuvieron evidencias de que la formación en emprendimiento aumenta la intención de emprender (Oosterbeek et al., 2010; Ruiz et al., 2014; Lima et al., 2015 y Popescu et al., 2016). También se rechaza la Hipótesis 2 (la educación emprendedora tiene un efecto positivo en la actitud ante el emprendimiento) debido a que la valoración media del alumnado decreció tras la formación (3,69 ex-antes, 3,56 expost).

Sin embargo, se han hallado evidencias que permiten afirmar que las Hipótesis 3 y 4 se sustentan. Concretamente, la valoración de control del comportamiento percibido ha aumentado significativamente tras la educación emprendedora (Hipótesis 3), es decir, el alumnado expresó que sus conocimientos para desarrollar y gestionar exitosamente una empresa se han elevado (2,76 ex-antes, 3,21 ex-post). Por ello, se coincide con Lanero et al. (2011), Nizam Zainuddin et al., (2012), Ruiz et al. (2014), Díaz-García et al. (2015) y Sánchez-García y Hernández-Sánchez (2016) al considerar que la educación emprendedora potencia el control del comportamiento percibido. Siguiendo a Joensuu-Salo et al. (2015), se considera que los programas de estudios y su metodología deben estar diseñados para que se gane confianza en las propias habilidades emprendedoras.

La estimación de las capacidades para emprender se ha incrementado, por ello la Hipótesis 4 se sustenta. Sucintamente, aumentó la valoración de: soy capaz de resolver prácticamente cualquier problema que se me presente; soy perfectamente capaz de desarrollar nuevos productos y servicios; $y$, soy perfectamente capaz de establecer una red de contactos personales y profesionales competencias y seguridad en sí mismo. En similitud con Sánchez-García y Hernández-Sánchez (2016), se afirma que no solo son necesarios conocimientos prácticos para emprender, sino que también es relevante poseer y desarrollar ciertas capacidades y competencias que aportaran de ventajas en la actividad emprendedora.

En cuanto a los efectos de la educación emprendedora en hombres y mujeres, se obtuvo evidencia de que la Hipótesis 5 (la educación emprendedora exhibe diferencias de género pues tiene un efecto superior en las mujeres que en los hombres) se sostiene. Ya que, solo para las alumnas la formación en emprendimiento implicó un aumento de la valoración de sus capacidades. El alumnado de la Universidad de Sevilla evidencia que la educación emprendedora tiene efectos diferentes en hombres y mujeres, repercutiendo cambios más profundos en ellas, al igual que autorías como Wilson et al. (2007) y Soria-Barreto, et al. (2017).

Los resultados expuestos deben hacer reflexionar tanto a investigadores como docentes para trabajar en el perfeccionamiento de los proyectos docentes. Asimismo, a través de la educación emprendedora se ha incrementado y mejorado el conocimiento teórico y práctico 
sobre el proceso emprendedor, lo cual consigue que el alumnado esté mejor formado y tenga más confianza sobre sus conocimientos. Pero esto no ha traído aparejado un aumento de la valoración de la intención emprendedora ni de la actitud personal ante el emprendimiento, es más, esta última variable se reduce. Conforme a ello, se piensa que es necesario profundizar en el análisis de las intenciones emprendedoras para conocer los motivos por los cuales se ha reducido la deseabilidad de crear una empresa. Autores como Díaz-García et al., (2015) y Caro-González et al. (2017) proponen incluir sesiones con emprendedores exitosos para que el alumnado conozca, experimente y se motive de primera mano con las vivencias emprendedoras. Estas personas transmitirán, mejor que nadie, los aspectos menos favorables y los más positivos del emprendimiento. Llevar a cabo dinámicas en el aula. Justamente, se coincide con Wilson (2009) al exponer la necesidad de plantearse modelos pedagógicos diferentes para mujeres y hombres, por lo que se puede incluir modelos referencia femeninos, con la intención de hacer más atractiva la carrera emprendedora. Además de orientar al profesorado a través de diferentes estilos de enseñanza y capacitación (Abaho, et al., 2015).

En consecuencia, se ha alcanzado el objetivo marcado, conocer cómo afecta la formación específica sobre creación de empresas a estudiantes del área de economía desde la perspectiva de género. A nivel teórico, esta investigación se adhiere a anteriores demostraciones, es decir, genera nuevas evidencias científicas sobre los temas educación y emprendimiento, en un ámbito en el que la literatura presenta carencias como es el sistema universitario andaluz.

Las implicaciones de la investigación involucran a dos principales destinatarios, la población joven estudiantil y la Administración Pública regional, pues se proporciona información de interés. Al igual que se contribuye a conocer las intenciones emprendedoras de los estudiantes actuales, futuros profesionales, se insta a la Administración Pública a reforzar el marco institucional que trabaje por el desarrollo y un futuro sólido.

Por otra parte, las limitaciones empíricas están ocasionadas por el tamaño de la muestra, por la acotación geográfica, temporal y económica, así como por el método de captación de datos. Señalar, también, que la información recogida a través de las encuestas se basa en una autoevaluación, por lo que es parcialmente información sesgada. En este sentido resultaría atractivo continuar la investigación incorporando en la medida de lo posible datos procedentes de otros métodos de captación de datos.

Así, futuros trabajos de investigación deberían considerar de interés comprobar si el perfil de los estudiantes de Economía obtenido se da en otros ámbitos geográficos y/o transciende a otras ramas de conocimientos. Incluso, se plantea estudiar la relación causal entre el perfil obtenido y los resultados académicos, al mismo tiempo, se propone continuar esta línea de trabajo en los cursos académicos venideros, obtener más temporalidades que permitan detallar la variación emprendedora en el ámbito estudiado. Igualmente, puede ampliarse la relación de la intención emprendedora con la tipología de enfoques, contenidos y metodologías docentes, así como su adecuación para desarrollarla (Ruiz et al., 2014). Siguiendo la propuesta de Medina et al. (2014, p. 77), se anhela abordar alguna pregunta para recabar información sobre el "tipo de formación emprendedora recibida por las personas universitarias y las carencias que ellas encuentran”. Igualmente, según Nabi et al. (2017, p. 277), se pueden usar "nuevos indicadores de impacto relacionados con la emoción y la mentalidad, el enfoque en los indicadores de impacto relacionados con la transición de intención de comportamiento". En último lugar, con respecto a los hallazgos relativos a la incidencia del género, los estudios indican que los modelos de rol tienen mayor influencia en las mujeres, y éstas parecen influir en la intención emprendedora, por tanto, parece necesario romper con los estereotipos reales. 


\section{REFERENCIAS}

Abaho, E., Olomi, D. R., y Urassa, G. C. (2015): “Students' entrepreneurial self-efficacy: does the teaching method matter?”, Education+ Training, vol.57, $\mathrm{n}^{\circ}$ 8/9, pp. 908-923. https://doi.org/10.1108/ET-02-2014-0008

Ajzen, I. (1991): "The theory of planned behavior", Organizational behavior and human decision processes, $\mathrm{n}^{0}$ 50, pp. 179-211. https://doi.org/10.1016/0749-5978(91)90020-T

Ajzen, I. (2005). Attitudes, personality, and behavior. McGraw-Hill Education (UK).

Bae, T, Qian, S, Miao, C and Fiet, J. (2014): “The Relationship between Entrepreneurship Education and Entrepreneurial Intentions: A Meta-Analytic Review", Entrepreneurship Theory and Practice, vol. 38, $\mathrm{n}^{\mathrm{o}}$ 2, pp. 217-254. https://doi.org/10.1111/etap.12095

Baptista, R., y Naia, A. (2015): "Entrepreneurship education: A selective examination of the literature", Foundations and Trends in Entrepreneurship, vol.11, n5, pp. 337-426. http://dx.doi.org/10.1561/0300000047

Bell, R. (2015): "Developing the next generation of entrepreneurs: Giving students the opportunity to gain experience and thrive”, The International Journal of Management Education, vol.13, nº1, pp. 37-47. https://doi.org/10.1016/j.ijme.2014.12.002

Boukamcha, F. (2015): "Impact of training on entrepreneurial intention: An interactive cognitive perspective”, European Business Review, vol.27, n ${ }^{\circ}$ 6, pp. 593-616. https://doi.org/10.1108/ EBR-12-2014-0090

Brink, T., y Madsen, S.O. (2015): "Entrepreneurial learning requires action on the meaning generated", International Journal of Entrepreneurial Behavior \& Research, vol. 21, $\mathrm{n}^{\mathrm{o}}$ 5, pp. 650-672, https://doi.org/10.1108/IJEBR-09-2014-0171

Camelo-Ordaz, C., Diánez-González, J. P., y Ruiz-Navarro, J. (2016): “The influence of gender on entrepreneurial intention: The mediating role of perceptual factors”, $B R Q$ Business Research Quarterly, vol. 19, n ${ }^{\circ} 4$, pp. 261-277. https://doi.org/10.1016/j.brq.2016.03.001

Caro-González, F. J., Romero-Benabent, H. A., y Sánchez-Torné, I. (2017): “The influence of gender on the entrepreneurial intentions of journalism students”, Intangible Capital, vol.13, nº 2, pp.430-478. http://dx.doi.org/10.3926/ic.927

Caro-González, F.J., Sánchez-Torné, I., Romero-Benabent, H. A (2018): "El modelo TCP aplicado a la formación en emprendimiento de los estudiantes en Comunicación”, Revista ESPACIOS. vol. 39, $\mathrm{n}^{\circ}$ 02. Recuperado de http://www.revistaespacios.com/a18v39n02/a18v39n02p07.pdf

Carrasco, A., Donoso, J.A., Duarte-Atoche, T., Hernández, J.J., y López, R. (2015): “Diseño y validación de un cuestionario que mide la percepción de efectividad del uso de metodologías de participación activa (CEMPA). El caso del Aprendizaje Basado en Proyectos (ABPrj) en la docencia de la contabilidad”, Innovar, vol.25, n58, pp.143. Disponible en https://www.redalyc.org/pdf/818/81841166011.pdf

Comisión Europea (2012). Entrepreneurship in the EU and Beyond. Report. Flash Eurobarometer, $n^{0} 354$.

Comisión Europea (2013). Plan de Acción sobre Emprendimiento 2020. Relanzar el espíritu emprendedor en Europa.

Davis, H.G., y Diamond, N. (1997): “The Rise of American Research Universities: Elites and Challengers in the Post-war Era”, Johns Hopkins University Press, Baltimore.

Díaz-Casero, J. C., Hernández-Mogollón, R., \& Barata-Raposo, M. L. (2007): “Estudiantes universitarios y creación de empresas. Un análisis comparativo entre España y Portugal”, En Conocimiento, innovación y emprendedores: camino al futuro. Universidad de La Rioja. pp. 1338-1355. 
Díaz-García, C., Sáez-Martínez, F., y Jiménez-Moreno, J. (2015): “Evaluación del impacto del programa educativo "Emprendedores" en la intención emprendedora de los participantes”, RUSC. Universities and Knowledge Society Journal, vol.12, $\mathrm{n}^{\circ} 3$. http://dx.doi.org/10.7238/rusc.v12i3.2146

Entrialgo, M., y Iglesias, V. (2016): "The moderating role of entrepreneurship education on the antecedents of entrepreneurial intention”, International Entrepreneurship and Management Journal, vol.12, nº 4, pp.1209-1232. https://doi.org/10.1007/s11365-0160389-4

ESIC Andalucía (2018). Estudio de Vocación Emprendedora en Universitarios Andaluces 2017. Ed. Andalucía Emprende, Fundación Pública Andaluza. Disponible en https://www.andaluciaemprende.es/wp-content/uploads/2017/11/Informe-EncuestaVocacio por cientoCC por ciento81n-Emprendedora-Universitarios-2017.pdf

Etzkowitz, H y Dzisah, J (2008): "Rethinking development: circulation in the triple helix", Technology Analysis and Strategic Management, vol. 20, pp. 653-666. https://doi.org/10.1080/09537320802426309

Fayolle, A., y Gailly, B. (2015): "The impact of entrepreneurship education on entrepreneurial attitudes and intention: Hysteresis and persistence”, Journal of Small Business Management, vol.53, $\mathrm{n}^{0}$ 1, pp.75-93. https://doi.org/10.1111/jsbm.12065

Fernández-Cornejo, J. A., Escot, L., Kabubo-Mariara, J., Kinyanjui Kinuthia, B., Eydal, G. B., y Bjarnason, T. (2016): “Gender differences in young adults' inclination to sacrifice career opportunities in the future for family reasons: comparative study with university students from Nairobi, Madrid, and Reykjavik”, Journal of Youth Studies, vol. 19, n 4, pp.457-482. https://doi.org/10.1080/13676261.2015.1083957

Fuentes-García, F. J., y Sánchez-Cañizares, S. M. (2010): “Análisis del perfil emprendedor: una perspectiva de género”, Estudios de Economía Aplicada, vol. 28, nº 3, pp. 1-27. Disponible en https://www.redalyc.org/pdf/301/30120334014.pdf

George, D., y Mallery, M. (2003). Using SPSS for Windows step by step: a simple guide and reference. Boston, MA: Allyn \& Bacon.

González, M. H., Valantina, I., Pérez, C., Aguado, S., Calabuig, F., y Crespo, J. J. (2016): “La influencia del género y de la formación académica en la intención de emprender de los estudiantes de ciencias de la actividad física y el deporte”, Intangible Capital, vol. 12, $\mathrm{n}^{\circ}$ 3, pp. 759-788. http://dx.doi.org/10.3926/ic.783

Goyanes, M. (2015): “Apoyo estructural en la intención emprendedora de estudiantes de periodismo y comunicación audiovisual en España”, El profesional de la información, vol.24, ${ }^{\circ} 1$, pp. 55-61. https://orcid.org/0000-0001-6537-9777

Hannon, P. (2006). Enterprise and Entrepreneurship in English Higher Education (Birmingham, NCGE).

Harrison, G.W., y List, J.A. (2004): "Field experiments", Journal of Economic literature, vol.42, nº4, pp.1009-1055. http://www.jstor.org/stable/3594915

Jaén, I., y Liñán, F. (2013): "Work values in a changing economic environment: the role of entrepreneurial capital”, International Journal of Manpower, vol. 34, n 8, pp.939-960. https://doi.org/10.1108/IJM-07-2013-0166

Jiang, H., Xiong, W., y Cao, Y. (2017): "Research on the mechanism of entrepreneurial education quality, entrepreneurial self-efficacy and entrepreneurial intention in social sciences, engineering and science education”, Eurasia Journal of Mathematics, Science \& Technology Education, vol. 13, $\mathrm{n}^{\mathrm{0}}$ 7, pp. 3709-3721. https://doi.org/10.12973/eurasia.2017.00754a

Joensuu-Salo, S., Varamäki, E., y Viljamaa, A. (2015): "Beyond intentions-what makes a student start a firm?”, Education+ Training, vol. 57, $\mathrm{n}^{\circ}$ 8/9, pp. 853-873. https://doi.org/10.1108/ET-11-2014-0142 
Jones, P., Jones, A., Packham, G., y Miller, C. (2008): "Student attitudes towards enterprise education in Poland: a positive impact”, Education+ Training, vol. 50, $\mathrm{n}^{\circ}$ 7, pp. 597614. https://doi.org/10.1108/00400910810909054

Krueger Jr., N.F., Reilly, M.D., y Carsrud, A.L. (2000): “Competing models of entrepreneurial intentions”, Journal of Business Venturing, vol. 15, $\mathrm{n}^{\circ}$ 5-6, pp.411-432. https://doi.org/10.1016/S0883-9026(98)00033-0

Krueger Jr., N.F., y Brazeal, D.V. (1994): "Entrepreneurial pot, ntial and potential entrepreneurs", Entrepreneurship Theory and Practice, vol. 18, $\mathrm{n}^{\circ}$ 3, pp. 91-104. https://doi.org/10.1177/104225879401800307

Krueger, A. O. (1993): "Virtuous and vicious circles in economic development", The American Economic Review, vol. 83, $\mathrm{n}^{\mathrm{o}}$ 2, pp. 351-355. http://www.jstor.org/stable/2117689

Lanero, A., Vázquez, J. L., Gutiérrez, P., y García, M. P. (2011): “The impact of entrepreneurship education in European universities: an intention-based approach analyzed in the Spanish area", International Review on Public and Nonprofit Marketing, vol. 8, n 2, pp. 111-130. https://doi.org/10.1007/s12208-011-0067-8

Lemmink, J., Schuijf, A., y Streukens, S. (2003): "The role of corporate image and company employment image in explaining application intentions”, Journal of Economic Psychology, vol. 24, no 1, pp.1-15. https://doi.org/10.1016/S0167-4870(02)00151-4

Lerís, D., y Sein-Echaluce, M.L. (2011): “La personalización del aprendizaje: Un objetivo del paradigma educativo centrado en el aprendizaje”, Arbor, n¹87(Extra_3), pp. 123-134. https://doi.org/10.3989/arbor.2011.Extra-3n3135

Lima, E., Lopes, R. M., Nassif, V., y Silva, D. (2015): "Opportunities to improve entrepreneurship education: Contributions considering brazilian challenges”, Journal of Small Business Management, vol. 53, $\mathrm{n}^{\mathrm{o}}$ 4, pp. 1033-1051. https://doi.org/10.1111/jsbm.12110

Liñán, F., Moriano, J. A., y Jaén, I. (2016): "Individualism and entrepreneurship: Does the pattern depend on the social context?”, International Small Business Journal, vol. 34, $n^{\circ}$ 6, pp.760-776. https://doi.org/10.1177/0266242615584646

Liñán, F., Rodríguez-Cohard, J. C., y Rueda-Cantuche, J. M. (2011): "Factors affecting entrepreneurial intention levels: a role for education”, International entrepreneurship and management Journal, vol. , no 2, pp. 195-218. https://doi.org/10.1007/s11365-0100154-z

Liñán, F., Urbano, D., y Guerrero, M. (2011): "Regional variations in entrepreneurial cognitions: start-up intentions of university students in Spain”, Entrepreneurship and Regional Development, vol. 23, $n^{\circ}$ 3-4, pp. 187-215. https://doi.org/10.1080/08985620903233929

Liñán, F., y Chen, Y. W. (2009): "Development and Cross-Cultural application of a specific instrument to measure entrepreneurial intentions", Entrepreneurship theory and practice, vol. 33, $\mathrm{n}^{\circ}$ 3, pp. 593-617. https://doi.org/10.1111/j.1540-6520.2009.00318.x

Liñán, F., y Fayolle, A. (2015): “A systematic literature review on entrepreneurial intentions: citation, thematic analyses, and research agenda”, International Entrepreneurship and Management Journal, vol. 11, nº 4, pp.907-933. https://doi.org/10.1007/s11365-0150356-5

Maresch, D., Harms, R., Kailer, N., y Wimmer-Wurm, B. (2016): "The impact of entrepreneurship education on the entrepreneurial intention of students in science and engineering versus business studies university programs”, Technological forecasting and social change, $\mathrm{n}^{\circ}$ 104, pp. 172-179. https://doi.org/10.1016/j.techfore.2015.11.006 
Maribel Guerrero, David Urbano, Antonio R. Ramos, José Ruiz-Navarro, Isabel Neira y Ana Fernández-Laviada. RedEmprendia y Centro Internacional Santander Emprendimiento (CISE) (2016). Perfil Emprendedor del Estudiante Universitario. Informe del Observatorio de Emprendimiento Universitario en España, 1, Edición 2015-2016. Madrid.

Márquez, E. y Jiménez-Rodrigo, M.L. (2014): "Project-based learning in virtual environments: a case study of a university teaching experience", Revista de Universidad y Sociedad del Conocimiento, vol. 11, $\mathrm{n}^{\mathrm{o}}$ 1, pp. 76-90. https://doi.org/10.7238/rusc.v11i1.1762

Martín, S., Fernández, J., Bel, P., y Lejarriaga, G. (2013): “Necesidad de medidas para impulsar la creación de las empresas de participación desde los diferentes niveles de enseñanza”, CIRIEC-España, Revista de Economía Pública, Social y Cooperativa, $\mathrm{n}^{\circ}$ 78, pp. 71-99. Disponible en https://www.redalyc.org/html/174/17429865006/

Medina, P., Bolívar, A., y Lemes, A.I. (2014): "Un paso más en la investigación de la intención emprendedora del estudiante universitario: GUESSS”, Revista de Estudios Empresariales. Segunda Época, n 2, pp. 63-80.

Moreira, M. A. (1993). Aprendizaje significativo: Un concepto subyacente. Recuperado de http://www. if. ufrgs. br/ moreira/apsigsubesp. pdf.

Mussons-Torras, M., y Tarrats-Pons, E. (2018): "Modelo de Credibilidad Emprendedora en los estudiantes de enfermería y fisioterapia”, Enfermería Global, vol. 17, n 49, pp. 294-323. http://dx.doi.org/10.6018/eglobal.17.1.280281

Nabi, G., Liñán, F., Fayolle, A., Krueger, N., y Walmsley, A. (2017): “The impact of entrepreneurship education in higher education: A systematic review and research agenda”. Academy of Management Learning \& Education, vol. 16, n² 2, pp. 277-299. https://doi.org/10.5465/amle.2015.0026

Nabi, G., Walmsley, A., Liñán, F., Akhtar, I., y Neame, C. (2018): "Does entrepreneurship education in the first year of higher education develop entrepreneurial intentions? The role of learning and inspiration”, Studies in Higher Education, vol. 43, n ${ }^{\circ} 3$, pp. 452467. https://doi.org/10.1080/03075079.2016.1177716

Naves, F., Cappelle, Alves, M., Angélico M.C., Souza, M. L., y Graças, M. (2012): "Teaching-learning critical perspective: tales of an experiment”, RAM. Revista de Administração Mackenzie, vol. 13, no 1, pp. 40-67. http://dx.doi.org/10.1590/S167869712012000100003

Ndofirepi, T. M., y Rambe, P. (2017): “Entrepreneurship education and its impact on the entrepreneurship career intentions of vocational education students”, Problems and Perspectives in Management, vol. 15, $\mathrm{n}^{\circ}$ 1-1, pp. 191-199.

Nizam Zainuddin, M., Fairuz Abd Rahim, M., y Rozaini Mohd Rejab, M. (2012): "Reconstruct creative destruction knowledge through creative disruption", On the Horizon, vol. 20, nº 1, pp. 34-48. https://doi.org/10.1108/10748121211202053

Olguín, E. y Caro-González, F.J. (2015): “A comprehensive ontological learning model for entrepreneurship training among engineering students", In 8th International Conference of Education, Research and Innovation (ICERI 2015) Proceedings, pp. 4182-4190.

Oliver, A., Galiana, L., y Gutiérrez-Benet, M. (2016): “Diagnóstico y políticas de promoción del emprendimiento en estudiantes”, Anales de Psicología, vol. 32, nº 1, pp. 183-189. http://dx.doi.org/10.6018/analesps.32.1.186681

Oosterbeek, H., Van Praag, M., y Ijsselstein, A. (2010): “The impact of entrepreneurship education on entrepreneurship skills and motivation”, European Economic Review, vol. 54, no 3, pp. 442-454. https://doi.org/10.1016/j.euroecorev.2009.08.002

Pihkala, J. (2008). Ammattikorkeakoulutuksen aikaiset yrittäjyysintentioiden muutokset. 
Disponible en http://tampub.uta.fi/handle/10024/67810

Piperopoulos, P., y Dimov, D. (2015): “·Burst bubbles or build steam? Entrepreneurship education, entrepreneurial self-efficacy, and entrepreneurial intentions”, Journal of Small Business Management, vol. 53, $\mathrm{n}^{\circ}$ 4, pp. 970-985. https://doi.org/10.1111/jsbm.12116

Popescu, C. C., Bostan, I., Robu, I. B., y Maxim, A. (2016): “An analysis of the determinants of entrepreneurial intentions among students: A Romanian case study”, Sustainability, vol. 8, n ${ }^{\circ}$ 8, pp. 771. http://dx.doi.org/10.3390/su8080771

Rauch, A., y Hulsink, W. (2015): "Putting entrepreneurship education where the intention to act lies: An investigation into the impact of entrepreneurship education on entrepreneurial behavior", Academy of Management Learning \& Education, vol. 14, $\mathrm{n}^{\circ}$ 2, pp. 187-204. https://doi.org/10.5465/amle.2012.0293

Romera-Iruela, M. J. (2011): "La investigación-acción en la formación del profesorado”, Revista Española de Documentación Científica, vol. 34, nº 4, pp. 597614. http://dx.doi.org/10.3989/redc.2011.4.836

Ruiz, C., García, F., y Delgado, N. (2014): “Condicionantes de la intención emprendedora en el alumnado universitario: un análisis desde la perspectiva de género”, Revista de Estudios Empresariales. Segunda Época, ${ }^{\circ}$ 2, pp. 81-96.

Ruiz-Navarro, J., Camelo-Ordaz, C. y Coduras-Martínez, A. (2012): "Mujer y desafío emprendedor en España: características y determinantes”, Economía Industrial, vol. 383, pp. 13-22.

Sahinidis, A. G., Giovanis, A. N., y Sdrolias, L. (2012): "The role of gender on entrepreneurial intention among students: An empirical test of the theory of planned behaviour in a Greek university”, International Journal on Integrated Information Management, vol. $1, \mathrm{n}^{\circ} 1$, pp. 61-79.

Salinas, F. y Osorio, L. (2012): "Emprendimiento y Economía Social, oportunidades y efectos en una sociedad en transformación”, CIRIEC-España, Revista de Economía Pública, Social y Cooperativa, $\mathrm{n}^{0} \quad 75, \quad$ pp. 129-151. Disponible en https://www.redalyc.org/html/174/17425798008/

Sánchez-García, J. C. (2010): "Evaluación de la personalidad emprendedora: validez factorial del cuestionario de orientación emprendedora (COE)", Revista Latinoamericana de Psicología, vol. 42, no 1, pp. 41-52.

Sánchez-García, J.C., Lanero, A., y Yurrebaso, A. (2005): "Determinants Variables of the Entrepreneurial Intention in the University Context”, Revista de Psicología Social Aplicada, vol. 15, n ${ }^{\mathrm{o}} 1$.

Sánchez-García, J.C., y Hernández-Sánchez, B. R. (2016): "Influencia del Programa Emprendedor Universitario (PREU) para la mejora de la actitud emprendedora", Pampa (Santa Fe), $\mathrm{n}^{\circ}$ 13, pp. 55-75.

Sanchis-Palacio, J. R., y Redondo-Cano, A. (1997): “Actitudes hacia la creación de empresas: estudio empírico aplicado a la Diplomatura de Relaciones Laborales de la Universitat de Valéncia”, CIRIEC-España, Revista de Economía Pública, Social y Cooperativa, ${ }^{\circ}$ 27, pp. 157-181.

Savery, J.R. (2015). Overview of problem-based learning: Definitions and distinctions, Essential Readings in Problem-Based Learning: Exploring and Extending the Legacy of Howard S. Barrows, pp. 5-15.

Shahverdi, M., Ismail, K., y Qureshi, M. (2018): “The effect of perceived barriers on social entrepreneurship intention in Malaysian universities: The moderating role of education", Management Science Letters, vol. 8, $\mathrm{n}^{\mathrm{o}}$ 5, pp. 341-352. 10.5267/j.msl.2018.4.014 
Shapero, A., y Sokol, L. (1982): “The social dimensions of entrepreneurship”. Encyclopedia of Entrepreneurship, pp. 72-90. Disponible en https://ssrn.com/abstract=1497759

Sieger, P., Gruber, M., Fauchart, E. y Zellweger T. (2016): "Measuring the Social Identity of Entrepreneurs: Scale Development and International Validation”, Journal of Business Venturing, vol. 31, no 5, pp. 542-572. https://doi.org/10.1016/j.jbusvent.2016.07.001

Soria-Barreto, K., Honores-Marín, G., Gutiérrez-Zepeda, P., y Gutiérrez-Rodríguez, J. (2017): "Prior Exposure and Educational Environment towards Entrepreneurial Intention". Journal of Technology Management \& Innovation, vol. 12, $\mathrm{n}^{\circ}$ 2, pp. 45-58. Disponible en https://scielo.conicyt.cl/pdf/jotmi/v12n2/art06.pdf

Srivastava, S., y Misra, R. (2017): "Exploring antecedents of entrepreneurial intentions of young women in India: A multi-method analysis”, Journal of Entrepreneurship in Emerging Economies, vol. 9, $\mathrm{n}^{\circ}$ 2, pp. 181-206. https://doi.org/10.1108/JEEE-042016-0012

Urbano, D., y Guerrero, M. (2013): "Entrepreneurial universities: Socioeconomic impacts of academic entrepreneurship in a European region”, Economic Development Quarterly, vol. 27, no 1, pp. 40-55. https://doi.org/10.1177/0891242412471973

Usman, B. (2019): "Understanding the entrepreneurial intention among international students in Turkey”. Journal of Global Entrepreneurship Research, vol. 9, $\mathrm{n}^{\mathrm{0}}$ 1, pp. 1-21. https://doi.org/10.1186/s40497-018-0136-0

Valencia, A., Montoya, I., y Montoya, A. (2016): “Intención emprendedora en estudiantes universitarios: Un estudio bibliométrico”, Intangible Capital, vol. 12, n 4 , pp. 881922. http://dx.doi.org/10.3926/ic.730

Valencia, A., Restrepo, I. A. M., y Restrepo, J. M. V. (2014): “Aportes teóricos y empíricos al estudio del emprendedor”, Cuadernos de Administración, vol. 30, nº 51, pp. 89-99. https://doi.org/10.25100/cdea.v30i51.46

Westhead, P., y Solesvik, M. Z. (2016): "Entrepreneurship education and entrepreneurial intention: do female students benefit?” International Small Business Journal, vol. 34, n ${ }^{\circ}$ 8, pp. 979-1003. https://doi.org/10.1177/0266242615612534

Wiek, A., Xiong, A., Brundiers, K., y Van Der Leeuw, S. (2014): “Integrating problem-and project-based learning into sustainability programs: A case study on the School of Sustainability at Arizona State University", International Journal of Sustainability in Higher Education, vol. 15, $\mathrm{n}^{\circ}$ 4, pp. 431-449. https://doi.org/10.1108/IJSHE-02-20130013

Wilson, F., Kickul, J., Marlino, D., Barbosa, S. D., y Griffiths, M. D. (2009): “An analysis of the role of gender and self-efficacy in developing female entrepreneurial interest and behavior”, Journal of developmental Entrepreneurship, vol. 14, n 2, pp. 105-119. https://doi.org/10.1142/S1084946709001247

Wilson, F., Kickul, J., y Marlino, D. (2007): "Gender, Entrepreneurial Self-Efficacy, and Entrepreneurial Career Intentions: Implications for Entrepreneurship Education”, Entrepreneurship Theory and Practice, vol. 31, $\mathrm{n}^{\circ}$ 3, pp. 387-406. https://doi.org/10.1111/j.1540-6520.2007.00179.x

WISE España, World Innovation Summit for Education, (2015): Encuesta: conectando la educación con el mundo real. Recuperado de https://www.wiseqatar.org/sites/default/files/asset/document/sp_inside-survey_201512.pdf

Zhao, H., Seibert, S. E., y Hills, G. E. (2005): "The mediating role of self-efficacy in the development of entrepreneurial intentions”, Journal of applied psychology, vol. 90, $\mathrm{n}^{\circ}$ 6, pp. 1265. http://dx.doi.org/10.1037/0021-9010.90.6.1265 